\title{
Loss of Progesterone Receptor Expression Is an Early Tumorigenesis Event Associated with Tumor Progression and Shorter Survival in Pancreatic Neuroendocrine Tumor Patients
}

Sung Joo Kim · Soyeon An Jae Hoon Lee ${ }^{1} \cdot$ Joo Young Kim² Ki-Byung Song ${ }^{1} \cdot$ Dae Wook Hwang ${ }^{1}$ Song Cheol Kim ${ }^{1}$. Eunsil Yu Seung-Mo Hong

\section{Department of Pathology and} 'Division of Hepatobiliary and Pancreatic Surgery, Asan Medical Center, University of Ulsan College of Medicine, Seoul; ' 2 Department of Pathology, Korea University Anam Hospital, Korea University College of Medicine, Seoul, Korea

Received: January 16, 2017

Revised: February 21, 2017

Accepted: March 19, 2017

Corresponding Author

Seung-Mo Hong, MD, PhD

Department of Pathology, Asan Medical Center,

University of Ulsan College of Medicine,

88 Olympic-ro 43-gil, Songpa-gu, Seoul 05505,

Korea

Tel: +82-2-3010-4558

Fax: $+82-2-472-7898$

E-mail: smhong28@gmail.com
Background: Pancreatic neuroendocrine tumors (PanNETs) are the second most common pancreatic neoplasms and there is no well-elucidated biomarker to stratify their detection and prognosis. Previous studies have reported that progesterone receptor (PR) expression status was associated with poorer survival in PanNET patients. Methods: To validate previous studies, PR protein expression was assessed in 21 neuroendocrine microadenomas and 277 PanNETs and compared with clinicopathologic factors including patient survival. Results: PR expression was gradually decreased from normal islets $(49 / 49$ cases, $100 \%)$ to neuroendocrine microadenoma (14/21, $66.6 \%)$ to PanNETs (60/277, 21.3\%; $p<.001)$. PanNETs with loss of PR expression were associated with increased tumor size $(p<.001)$, World Health Organization grade $(p=.001)$, pT classification $(p<$ $.001)$, perineural invasion $(p=.028)$, lymph node metastasis $(p=.004)$, activation of alternative lengthening of telomeres $(p=.005)$, other peptide hormonal expression $(p<.001)$ and ATRX/DAXX expression $(p=.015)$. PanNET patients with loss of PR expression (5-year survival rate, $64.1 \%$ ) had significantly poorer recurrence-free survival outcomes than those with intact PR expression $(90 \%)$ by univariate $(p=.012)$ but not multivariate analyses. Similarly, PanNET patients with PR expression loss (5-year survival rate, $76 \%)$ had significantly poorer overall survival by univariate $(p=.015)$ but not multivariate analyses. Conclusions: Loss of PR expression was noted in neuroendocrine microadenomas and was observed in the majority of PanNETs. This was associated with increased grade, tumor size, and advanced $\mathrm{pT}$ and pN classification; and was correlated with decreased patient survival time by univariate but not multivariate analyses. Loss of PR expression can provide additional information on shorter disease-free survival in PanNET patients.

Key Words: Pancreas; Neuroendocrine tumors; Receptors, progesterone; Survival
Pancreatic neuroendocrine tumors (PanNETs) are rare, amounting to only $3 \%$ of pancreatic neoplasms ${ }^{1,2}$ and $9 \%$ of all gastroenteropancreatic neuroendocrine tumors in Korea. ${ }^{3}$ Although PanNET patients have better survival outcomes than pancreatic ductal adenocarcinoma patients, PanNETs are still malignant neoplasms with a 10-year survival rate of only $40 \%-$ $50 \%$ after surgical resection. ${ }^{4-6}$ Surgical resection is the main curative treatment option for PanNET patients, although other therapies with somatostatin analogs, cytotoxic chemotherapies, and molecular targeted therapies have recently been used in metastatic or unresectable PanNET patients. ${ }^{7,8}$

A better understanding of the molecular mechanisms of PanNETs is important to better predict clinical outcomes and identify patients who may benefit from therapies targeting PanNETs. A previous whole-exome sequencing study revealed the genomic landscape of PanNETs including a higher mutational frequency in MEN1, ATRX (alpha thalassemia/mental retardation syndrome $\mathrm{X}$-linked), and $D A X X$ (death-domain associated protein) and a lower mutational frequency in several genes involving mammalian target of rapamycin pathways, including PTEN, TSC2, and PIK3CA. ${ }^{9}$ In that study also, less than half of PanNETs had inactivating mutations in either the ATRX or DAXX genes, in mutually exclusive ways. ${ }^{9}$ Alterations in the ATRX or DAXX proteins were closely associated with the alternative lengthening of telomeres (ALT) mechanism in PanNETs, ${ }^{10}$ and the loss of ATRX or DAXX expression and ALT activation were associated with poorer survival in primary PanNET patients. ${ }^{11-13}$

The hormonal expression status in PanNETs has clinical im- 
plications. Peptide hormonal expression is reported to have better prognostic implications for PanNET patients. Increased expression of insulin, GLP1, and other peptide hormones are associated with better overall survival in PanNET patients, whereas gastrin expression has been reported to be associated with worse survival. ${ }^{14}$ Among the steroid hormones, only progesterone receptors (PRs), but not estrogen receptors or androgen receptors, are expressed in normal islets. ${ }^{15-17} \mathrm{PR}$ expression in normal pancreatic islets suggests a possible role for progesterone in pancreatic islet function. A study in mice with intact gonads demonstrated that progesterone treatment stimulated $\beta$-cell proliferation in pancreatic islets. ${ }^{1}$ However, those proliferation effects were lost in gonadectomized mice. Those observations suggested that progesterone effects require intact gonadal function for normal $\beta$-cell proliferation. ${ }^{1}$ In our present study, we determined the clinical and prognostic significances of $\mathrm{PR}$ expression in surgically resected PanNET patients using tissue microarray immunolabeling.

\section{MATERIALS AND METHODS}

\section{Case selection}

After approval (2015-0387) from the Institutional Review Board of Asan Medical Center, the records of 277 surgically resected primary PanNETs and 21 sporadic neuroendocrine microadenomas resected between January 1995 and December 2015 were retrieved from the Department of Pathology at Asan Medical Center. Primary PanNETs were defined as well-differentiated, nonfunctional neuroendocrine neoplasms with diameters $\geq 0.5 \mathrm{~cm}$, while neuroendocrine microadenomas were defined as well-differentiated, nonfunctional NETs with diameters $<0.5 \mathrm{~cm} .{ }^{1,18}$ All PanNET cases were classified using the 2010 World Health Organization (WHO) classification scheme with mitotic activity and the Ki67 labeling index. ${ }^{1}$ The Ki-67 labeling index was measured by manually assessing the tumor's hottest spot in high-power fields after printing the captured image, as previously described. ${ }^{19} \mathrm{~A}$ minimum of 500 tumor cells were included in the manual count. Poorly differentiated neuroendocrine carcinomas, such as small-cell carcinomas and large-cell carcinomas, were excluded. Pathological data, such as tumor size, extension, lymph node and distant metastases, and perineural and lymphovascular tumor invasion, were extracted from the pathology reports. The clinical data reviewed included patient age, gender, symptoms, and survival outcomes. The expression profiles of specific peptide hormones, including insulin, glucagon, gastrin, serotonin, somatostatin, and glucagon-like peptide 1; other proteins, such as ATRX and DAXX; and the ALT status were used as previously reported. ${ }^{12,14}$

\section{Tissue microarray construction}

Tissue microarrays were constructed from archived, formalinfixed, paraffin-embedded tissue blocks with a manual tissue microarrayer (Uni TMA Co. Ltd., Seoul, Korea), as previously described. ${ }^{12,14,20}$ Briefly, three cores from the tumors and one core from the normal pancreatic parenchyma with a diameter of $2 \mathrm{~mm}$ were punched from donor blocks and placed in recipient blocks.

\section{Immunohistochemical staining}

Immunohistochemical labeling was performed at the immunohistochemical laboratory of the Department of Pathology, Asan Medical Center. In brief, 4-mm-thick tissue sections were deparaffinized and hydrated in xylene and serially diluted in ethanol. Endogenous peroxidase was blocked by incubation in $3 \% \mathrm{H}_{2} \mathrm{O}_{2}$ for 10 minutes, and then heat-induced antigen retrieval was performed. Primary antibodies were used with a Benchmark autostainer (Ventana Medical Systems, Tucson, AZ, USA) in accordance with the manufacturer's protocol. Sections were incubated at room temperature for 32 minutes in primary antibodies for PR (1:200, NCL-L-PGR-312, Novocastra, Newcastle upon Tyne, UK), synaptophysin (1:200, DiNona, Seoul, Korea), chromogranin (1:200, DAK-A3, DakoCytomation, Glostrup, Denmark), and Ki-67 (1:100, 7B11, Zymed, San Francisco, CA, USA). The sections were then labeled with an automated immunostaining system and processed with an iView DAB detection kit (Benchmark XT, Ventana Medical Systems). Immunostained sections were lightly counterstained with hematoxylin, dehydrated in ethanol, and cleared in xylene. Immunoreactivity was interpreted by light microscopic examination and independently evaluated by two pathologists, coauthors of this study (S.J.K. and S.M.H.), who were blind to the clinicopathologic information.

\section{Evaluation of PR labeling}

Immunohistochemical labeling of the PR protein was scored using a previously described histological scoring system, which takes into account the size of the stained area and the intensity of the labeling. To be included in the analyses, a tumor had to have sufficient numbers of PR-labeled cells to permit quantification (> 100 PR-positive tumor cells). The labeled area was scored from 0 to 4 for having < 5\%, 5\%-25\%, 26\%-50\%, $51 \%-75 \%$, or $>75 \%$ PR-positive cells, respectively. The intensity scale ranged from 0 to 2 as follows: 0 , no labeling of tumor cells; 1 , weak labeling; and 2 , intense labeling, as previously described. ${ }^{21}$ 
The total histological score (H-score) was calculated by multiplying the area score by the intensity score. The resulting $\mathrm{H}$-score ranged from 0 to 8 . We considered cases with an $\mathrm{H}$-score below 2 to have loss of PR expression and cases with an $\mathrm{H}$-score greater than or equal to 2 to have intact PR expression. PR labeling in normal islets was used as an internal positive control. Representative images of PR expression in normal islet and in PanNETs are depicted in Fig. 1. Immunohistochemical staining was evaluated by two independent pathologists (S.J.K. and S.M.H.).

\section{Statistical analyses}

SPSS software ver. 20.0 (IBM Corp., Chicago, IL, USA) was used for statistical analyses. The overall and recurrence-free survival times were calculated from the date of diagnosis of PanNET to that of death from any cause and from the date of diagnosis of PanNET to that of recurrence, respectively. Both overall and recurrence-free survival rates were calculated using the KaplanMeier method, and the association between overall survival rate and clinicopathologic factors was compared using the log-rank

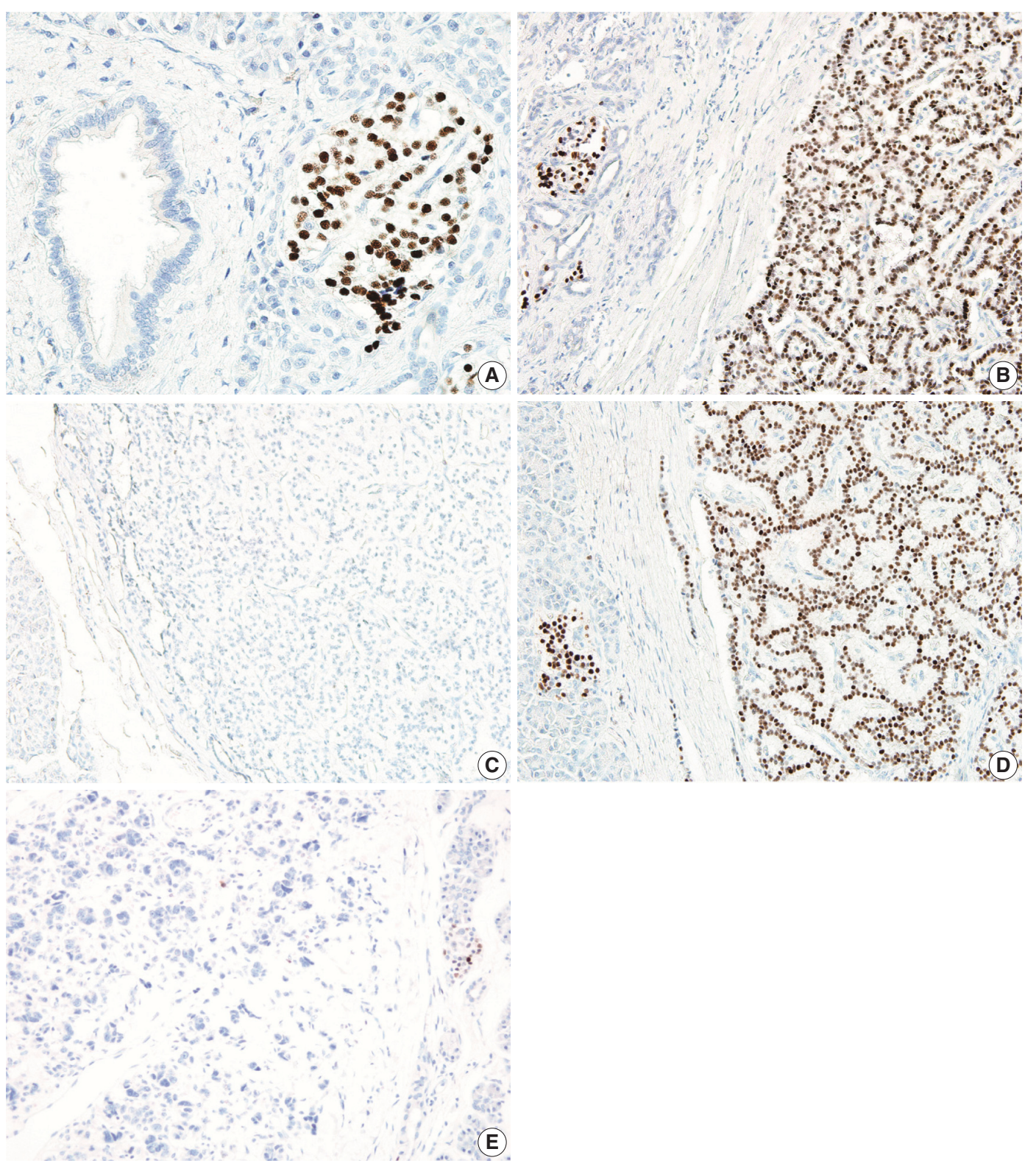

Fig. 1. Representative images of progesterone receptor (PR) labeling in normal pancreas, neuroendocrine microadenoma, and pancreatic neuroendocrine tumor (PanNET). (A) Islets are positive, while acinar and ductal epithelial cells are negative for PR staining in the normal pancreas. Some neuroendocrine microadenomas show intact PR labeling $(B)$, while other neuroendocrine microadenomas demonstrate loss of PR labeling (C). Some PanNETs show intact PR labeling (D), while other PanNETs demonstrate loss of PR labeling (E). 
test. The correlations between PR expression and other prognostic factors were analyzed using the chi-square and Fisher exact tests. Possible prognostic factors associated with survival probability were calculated using the Cox's proportional hazard regression model; $\mathrm{p}<.05$ was considered statistically significant.

\section{RESULTS}

\section{Patient characteristics}

Patient characteristics are summarized in Table 1. In the total cohort, 134 patients (49\%) were male and $143(51 \%)$ were female. The mean age of the patients was $52.3 \pm 12.7$ years. According to the WHO classification, there were $85 \mathrm{G} 1,95 \mathrm{G} 2$, and $8 \mathrm{G} 3$, respectively. The mean tumor size of the PanNETs and neuroendocrine microadenomas was $3.0 \pm 2.2 \mathrm{~cm}$ and $0.3 \pm 0.1 \mathrm{~cm}$, respectively. In all, 126 cases were classified as pT1, while others had higher pT classifications (95 pT2, 52 pT3, and 4 pT4); 79 cases $(28.5 \%)$ had lymphovascular invasion and 42 cases (15.2\%) had perineural invasion. Metastasis to regional lymph nodes occurred in 39 cases $(14.1 \%)$ and metastasis to distant organs at the surgical resection of PanNET was observed in 10 cases (3.6\%). The median follow-up period was $38 \pm 35$ months (range, 1 to 188 months).

\section{PR expression}

All of the normal pancreatic islets in the patient samples expressed the PR protein in various proportions. PR protein expression was observed in $63.5 \pm 9.8 \%$ of the endocrine cells in the islets. A representative image of PR expression in an islet is depicted in Fig. 1. This expression gradually decreased from normal islets $(49 / 49,100 \%)$ to neuroendocrine microadenoma (14/21, 66.6\%) to PanNETs (60/277, 21.3\%). The H-scores for PR in normal islets, neuroendocrine microadenomas, and PanNETs were $5.1 \pm 2.3,3.0 \pm 3.3$, and $1.3 \pm 2.5$, respectively ( $\mathrm{p}<.001$ ) (Figs. $1,2)$. The mean $\mathrm{H}$-score of the PR expression loss group was 0.1 \pm 0.3 , while that of the PR expression intact group was $5.8 \pm$ 2.3. The majority of PanNETs showed a loss of PR expression (218/277, 78.7\%) (Fig. 1).

\section{Correlations between PR expression and clinicopathologic factors}

The associations between PR expression and clinicopathologic factors are summarized in Table 1. Loss of PR expression was more commonly observed in PanNETs with larger tumors $(\mathrm{p}<$ $.001)$, a higher WHO grade $(\mathrm{p}=.001)$, higher Ki-67 labeling index $(\mathrm{p}=.004)$, higher $\mathrm{pT}$ classification $(\mathrm{p}<.001)$, frequent peri- neural invasion $(\mathrm{p}=.028)$, and regional lymph node metastasis $(\mathrm{p}=$ .004). Loss of PR expression was also strongly associated with other peptide hormonal expression $(\mathrm{p}<.001)$, loss of ATRX/ DAXX expression ( $p=.015)$, and activation of ALT ( $p=.005)$. In addition, the loss of PR expression was marginally associated with lymphovascular invasion $(\mathrm{p}=.077)$ but not with age, gender, or distant metastasis.

Table 1. Clinicopathologic factors associated with PR expression in PanNETs

\begin{tabular}{|c|c|c|c|}
\hline Characteristic & PR loss & Intact PR & p-value \\
\hline Age (yr) & & & .412 \\
\hline$\leq 60$ & 149 (78.0) & $42(22)$ & \\
\hline$>60$ & $69(80.2)$ & $17(19.8)$ & \\
\hline Sex & & & .380 \\
\hline Male & 107 (79.9) & $27(20.1)$ & \\
\hline Female & $111(77.6)$ & $32(22.4)$ & \\
\hline Tumor size $(\mathrm{cm})$ & & & $<.001$ \\
\hline$\leq 3$ & $126(71.2)$ & $51(28.8)$ & \\
\hline$>3$ & $92(86.4)$ & $8(13.6)$ & \\
\hline WHO grade & & & .001 \\
\hline Grade 1 & $65(76.5)$ & 20 (23.5) & \\
\hline Grade 2 & $83(87.4)$ & $12(12.6)$ & \\
\hline Grade 3 & $8(100.0)$ & 0 & \\
\hline pT classification & & & $<.001$ \\
\hline pT1 & $70(62.5)$ & $42(27.5)$ & \\
\hline pT2-T4 & $148(89.7)$ & $17(10.3)$ & \\
\hline Lymphovascular invasion & & & .077 \\
\hline Absent & $151(76.3)$ & $47(23.7)$ & \\
\hline Present & $67(84.8)$ & $12(15.2)$ & \\
\hline Perineural invasion & & & .028 \\
\hline Absent & $180(76.6)$ & $55(23.4)$ & \\
\hline Present & $38(90.5)$ & $4(9.5)$ & \\
\hline Lymph node metastasis & & & .004 \\
\hline Absent & $181(76.1)$ & $57(23.9)$ & \\
\hline Present & $37(94.9)$ & $2(5.1)$ & \\
\hline Distant metastasis & & & .639 \\
\hline Absent & $210(78.7)$ & 57 (21.3) & \\
\hline Present & $8(80.0)$ & $2(20.0)$ & \\
\hline Ki-67 labeling index (\%) & & & .004 \\
\hline$<3$ & $137(73.3)$ & $50(26.7)$ & \\
\hline$\geq 3$ and $<20$ & 57 (89.1) & 7 (10.9) & \\
\hline$\geq 20$ & $2(100.0)$ & 0 & \\
\hline Hormone expression & & & $<.001$ \\
\hline Absent & $91(94.8)$ & $5(5.2)$ & \\
\hline Present & $49(68.1)$ & $23(31.9)$ & \\
\hline ALT expression & & & .005 \\
\hline Absent & $166(75.7)$ & $54(24.5)$ & \\
\hline Present & $52(91.2)$ & $5(8.8)$ & \\
\hline ATRX/DAXX expression & & & .015 \\
\hline Absent & $45(90.0)$ & $5(10.0)$ & \\
\hline Present & $166(75.5)$ & $54(24.5)$ & \\
\hline
\end{tabular}

Values are presented as number (\%).

PR, progesterone receptor; PanNET, pancreatic neuroendocrine tumor; WHO, World Health Organization; ALT, alternative lengthening of telomeres. 

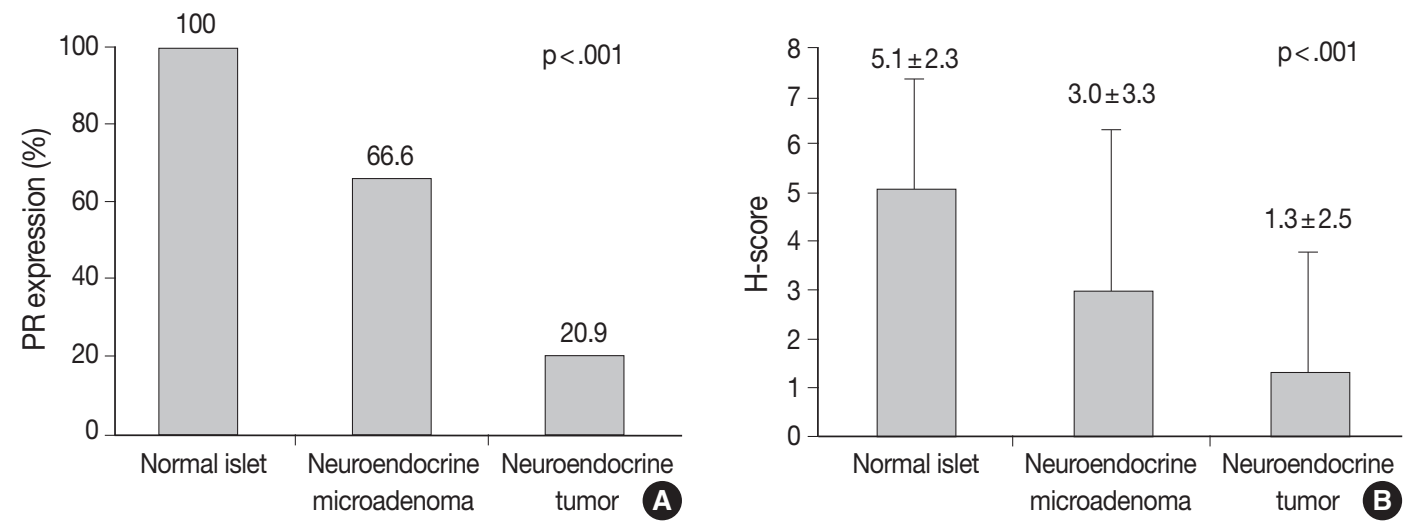

Fig. 2. Progesterone receptor (PR) expression status and the $\mathrm{H}$-score in normal islets, neuroendocrine microadenomas, and pancreatic neuroendocrine tumors (PanNETs). (A) Comparison of PR expression in normal islets, neuroendocrine microadenomas, and PanNETs. (B) The $\mathrm{H}$-score for PR in normal islets, neuroendocrine microadenomas, and PanNETs is $5.1 \pm 2.3,3.0 \pm 3.3$, and 1.3 \pm 2.5 , respectively ( $p<.001)$.
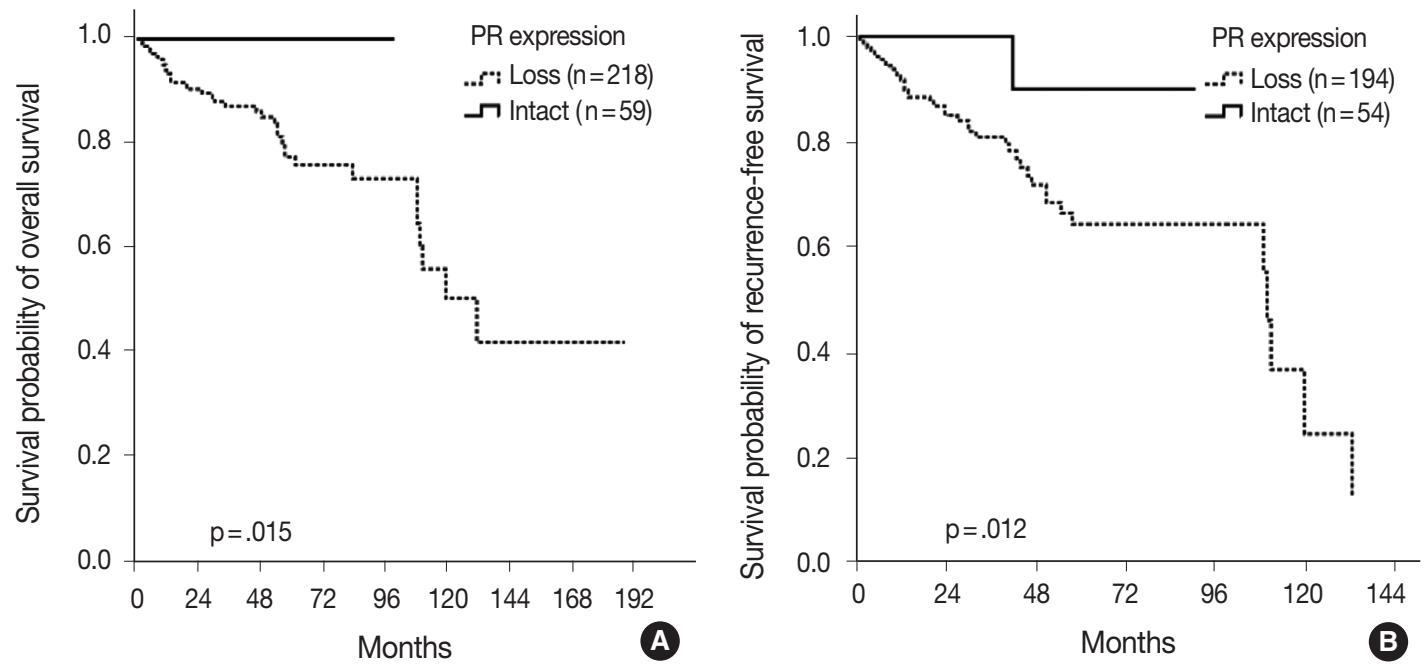

Fig. 3. Kaplan-Meier survival analyses of pancreatic neuroendocrine tumor (PanNET) patients according to progesterone receptor (PR) expression. (A) The 100\% overall 5-year survival rate for PanNET patients with PR expression is significantly better than that for those without insulin expression (76\%, $p=.015)$. (B) The recurrence-free 5-year survival rate for PanNET patients with PR expression (90\%) is significantly better than that for those without PR expression $(64.1 \%, p=.012)$.

\section{Survival analyses of PR expression}

The overall 5-year survival rate of $100 \%$ among the PanNET patients with intact PR expression was significantly better than for patients with PR expression loss $(76 \%, \mathrm{p}=.015)$ (Fig. 3A). Similarly, the recurrence-free 5-year survival rate in the PanNET patients with intact PR expression (90\%) was significantly better than that of those with PR expression loss $(64.1 \%, \mathrm{p}=.012)$ (Fig. 3B).

\section{Univariate analyses of other clinicopathologic factors}

The relationships found between survival and other clinicopathologic factors are summarized in Table 2. The clinicopathologic factors associated with poorer survival, according to univariate survival analyses, were older age $(\mathrm{p}=.015)$, larger tumor size $(\mathrm{p}=$
.008), higher WHO grade ( $\mathrm{p}<.001)$, higher $\mathrm{pT}$ classification $(\mathrm{p}=$ $.015)$, and frequent lymphovascular $(\mathrm{p}=.002)$, perineural $(\mathrm{p}<$ $.001)$, and regional lymph node metastasis $(\mathrm{p}<.001)$.

\section{Multivariate analyses of clinicopathologic factors}

The Cox proportional hazard model was employed with other significant clinicopathologic factors to determine the prognostic significance of PR expression as well as other clinicopathologic factors in PanNET patients. Only WHO grade $(\mathrm{p}=.001)$ and lymphovascular invasion ( $\mathrm{p}=.020)$ were independently prognostic, but loss of PR expression was not a prognostic factor in our model ( $\mathrm{p}=$ .117) (Table 2). 


\section{DISCUSSION}

PR immunohistochemistry has been used to identify ovariantype stroma for the diagnosis of mucinous cystic neoplasms of the pancreas in the field of pancreas pathology. ${ }^{18} \mathrm{~A}$ recent study demonstrated that loss of or decreases in PR expression in ovarian type stroma of invasive carcinoma with mucinous cystic neoplasm and mucinous cystic neoplasm with high-grade dysplasia were associated with decreased volumes of ovarian-type stroma in mucinous cystic neoplasms. ${ }^{22}$ These results suggest the possible utility of PR immunolabeling as a surrogate marker for invasion in the diagnosis of mucinous cystic neoplasms.

In addition to the use of PR expression for diagnosing mucinous cystic neoplasms, this expression has also been reported in all normal islets of Langerhans, and previous studies have detected PR in 40\%-75\% of islets. ${ }^{16,17}$ In our current study, PR expression was noted in $64 \%$ of normal islets, a similar proportion to that found in previous studies.

Neuroendocrine microadenomas are precursors and initiating lesions for PanNETs. ${ }^{23,24}$ Only a few previous studies have conducted biomarker evaluations for neuroendocrine microadenomas. ${ }^{12,25,26}$ Decreased menin expression and increased cytokeratin 19 expression have been reported in neuroendocrine microadenomas. ${ }^{25,26}$ However, controversy exists over the loss of ATRX or DAXX expression in these tumors. ${ }^{12,26,27}$ Confirming previous findings, we observed PR expression in 67\% of neuroendocrine microadenomas, and our observations suggest that PR expression loss can also be included as an early event in neuroendocrine tumorigenesis.

PR expression was present in only $21 \%$ of the PanNETs examined in our current study, and the majority of PanNETs showed loss of PR expression. Previous studies have found wide ranges of PR expression, from $46 \%$ to $76 \% .^{16,28,29}$ Plausible explanations for the lower PR expression in our study include different ethnic backgrounds (Western vs Korean), antibody clones, and the cutoff point used for positive expression. While previous studies examined European or American populations, the present study was performed on a Korean population. This difference in ethnic background could have affected PR expression. In addition, previous studies considered PR expression loss when the proportion of nuclear PR labeling was $<1 \%$ or $<5 \%,{ }^{16,28,29}$ whereas we used the $\mathrm{H}$-score, multiplying the intensity and proportion of PR expression. Different cutoff points for the evaluation of PR expression could explain the lower levels detected in our study.

Loss of PR expression was associated with larger tumor size, higher WHO grade, higher $\mathrm{pT}$ classification, and frequent lymphovascular and perineural invasion, and regional lymph node metastasis. Our current observations are thus concordant with the results of previous studies. ${ }^{16,28}$ Arnason et al..$^{15}$ previously examined 40 PanNET cases and observed that PanNETs with strong PR expression were associated with fewer nodal or distant metastases of PanNETs. Viale et al. ${ }^{16}$ studied 96 PanNETs and reported that less

Table 2. Univariate and multivariate analyses of PR expression in PanNETs

\begin{tabular}{|c|c|c|c|c|c|c|}
\hline \multirow{2}{*}{ Characteristic } & \multirow{2}{*}{ Variables } & \multirow{2}{*}{$\begin{array}{c}\text { Univariate analyses } \\
5 \text {-Year survival rate (\%) }\end{array}$} & \multirow{2}{*}{ p-value } & \multicolumn{2}{|c|}{ Multivariate analyses } & \multirow{2}{*}{ p-value } \\
\hline & & & & Hazard ratio & $95 \%$ confidence interval & \\
\hline \multirow[t]{2}{*}{ PR expression } & Loss & 64.1 & .012 & 0.20 & $0.03-1.49$ & .117 \\
\hline & Intact & 90 & & & & \\
\hline \multirow[t]{2}{*}{ Age (yr) } & $\leq 60$ & 70.3 & .015 & 1.58 & $0.76-3.23$ & .228 \\
\hline & $>60$ & 62.1 & & & & \\
\hline Sex & Female & 56.9 & & & & \\
\hline \multirow[t]{2}{*}{ Tumor size (cm) } & $\leq 3$ & 74.6 & .008 & 0.94 & $0.37-2.39$ & .879 \\
\hline & $>3$ & 55.7 & & & & \\
\hline \multirow[t]{3}{*}{ WHO grade } & Grade 1 & 76.9 & $<.001$ & 1.00 & - & .001 \\
\hline & Grade 2 & 65.8 & & 1.24 & $0.60-2.56$ & .558 \\
\hline & Grade 3 & 0 & & 10.74 & $3.79-30.45$ & $<.001$ \\
\hline pT classification & pT2-T4 & 61.8 & & & & \\
\hline \multirow[t]{2}{*}{ Lymphovascular invasion } & Absent & 71.5 & .002 & 2.19 & $1.13-4.23$ & .020 \\
\hline & Present & 54 & & & & \\
\hline \multirow[t]{2}{*}{ Perineural invasion } & Absent & 71.5 & $<.001$ & 1.12 & $0.40-3.16$ & .813 \\
\hline & Present & 41 & & & & \\
\hline \multirow[t]{2}{*}{ Lymph node metastasis } & Absent & 73 & $<.001$ & 1.65 & $0.60-4.55$ & .341 \\
\hline & Present & 27.8 & & & & \\
\hline
\end{tabular}

PR, progesterone receptor; PanNET, pancreatic neuroendocrine tumor; WHO, World Health Organization. 
PR immunoreactivity was more commonly associated with malignant behaviors, including metastasis, invasion of surrounding tissues, or larger vessel involvement. Estrella et al..$^{28}$ reviewed 160 PanNET cases and found that loss of PR expression was associated with larger tumor size and advanced American Joint Committee on Cancer tumor staging but was not correlated with age, gender, or WHO grade.

In our present study series, we observed that PanNET patients with PR expression loss had significantly poorer overall and disease-free survival outcomes by univariate but not multivariate analyses. Thus, PR status can provide additional survival information for PanNET patients but cannot be used as a prognostic indicator. Previous studies have also evaluated PanNETs with PR expression and patient survival. ${ }^{15,28}$ Arnason $e t$ al. ${ }^{15}$ reported that pancreas and small intestinal NET patients with intact PR expression had significantly better disease-free survival (median, 155 months) than those with decreased PR expression (median, 38 months). However, they further found that this was only marginally significant when they restricted their examination to only PanNET patients. ${ }^{15}$ Estrella et al. ${ }^{28}$ observed no significant differences in overall survival based on PR expression status only. However, when they compared the survival of PanNET patients after combining PTEN and PR expression status, dual PR- and PTEN-negative PanNET patients showed shorter metastasisfree survival than either single PR- or PTEN-positive patients or dual PR-and PTEN-positive patients. ${ }^{28}$

The biological roles of PR in normal islets and PanNETs have not been completely elucidated. One previous study demonstrated that the administration of progesterone to PR knock-out mice with intact gonads induced $\beta$-cell proliferation, which suggests antiproliferation activity for PR. ${ }^{2}$ PRs exists as two protein isoforms, PRA and PRB. ${ }^{30}$ Recently, Yazdani et al..$^{31}$ demonstrated that PanNET tumorigenesis occurred via activation of PRB after its binding to progesterone, which was induced by the activation of transcription factors FOS and Jun and followed by overexpression of CCND1. They also demonstrated that PRA in the progesterone signaling pathway inhibited PanNET tumorigenesis by suppressing the PRB promoter. ${ }^{31}$

In summary, we performed an immunohistochemical study of PR in 21 surgically resected neuroendocrine microadenomas and 277 PanNETs. Our key findings were loss of PR expression in neuroendocrine microadenomas and in the majority of PanNETs and associated with increased WHO grade, tumor size, and advanced $\mathrm{pT}$ and $\mathrm{pN}$ classification. The loss of $\mathrm{PR}$ expression also correlated with decreased patient survival time according to univariate, but not multivariate, analysis. In conclusion, the loss of
PR expression can provide additional information on shorter diseasefree survival outcomes in PanNET patients.

\section{Conflicts of Interest}

No potential conflict of interest relevant to this article was reported.

\section{Acknowledgments}

This work was supported by the Basic Science Research Program through the National Research Foundation of Korea (NRF) funded by the Ministry of Education, Science and Technology (2016R1A2B4009381) and a grant (2013-554) from the Asan Institute for Life Sciences, Seoul, Republic of Korea.

\section{REFERENCES}

1. Bosman FT, Carneiro F, Hruban RH, Theise ND. WHO classification of tumours of the digestive system. 4th ed. Lyon: IARC Press, 2010.

2. Picard F, Wanatabe M, Schoonjans K, Lydon J, O'Malley BW, Auwerx J. Progesterone receptor knockout mice have an improved glucose homeostasis secondary to beta -cell proliferation. Proc Natl Acad Sci US A 2002; 99: 15644-8.

3. Gastrointestinal Pathology Study Group of Korean Society of Pathologists, Cho MY, Kim JM, et al. Current trends of the incidence and pathological diagnosis of gastroenteropancreatic neuroendocrine tumors (GEP-NETs) in Korea 2000-2009: multicenter study. Cancer Res Treat 2012; 44: 157-65.

4. Bilimoria KY, Talamonti MS, Tomlinson JS, et al. Prognostic score predicting survival after resection of pancreatic neuroendocrine tumors: analysis of 3851 patients. Ann Surg 2008; 247: 490-500.

5. Fesinmeyer MD, Austin MA, Li CI, De Roos AJ, Bowen DJ. Differences in survival by histologic type of pancreatic cancer. Cancer Epidemiol Biomarkers Prev 2005; 14: 1766-73.

6. Halfdanarson TR, Rabe KG, Rubin J, Petersen GM. Pancreatic neuroendocrine tumors (PNETs): incidence, prognosis and recent trend toward improved survival. Ann Oncol 2008; 19: 1727-33.

7. Kulke $\mathrm{MH}$, Shah $\mathrm{MH}$, Benson $\mathrm{AB} 3 \mathrm{rd}$, et al. Neuroendocrine tumors, version 1.2015. J Natl Compr Canc Netw 2015; 13: 78-108.

8. Amin S, Kim MK. Islet cell tumors of the pancreas. Gastroenterol Clin North Am 2016; 45: 83-100.

9. Jiao Y, Shi C, Edil BH, et al. DAXX/ATRX, MEN1, and mTOR pathway genes are frequently altered in pancreatic neuroendocrine tumors. Science 2011; 331: 1199-203.

10. Heaphy CM, de Wilde RF, Jiao Y, et al. Altered telomeres in tumors 
with ATRX and DAXX mutations. Science 2011; 333: 425.

11. Marinoni I, Kurrer AS, Vassella E, et al. Loss of DAXX and ATRX are associated with chromosome instability and reduced survival of patients with pancreatic neuroendocrine tumors. Gastroenterology 2014; 146: 453-60.

12. Kim JY, Brosnan-Cashman JA, An S, et al. Alternative lengthening of telomeres in primary pancreatic neuroendocrine tumors is associated with aggressive clinical behavior and poor survival. Clin Cancer Res 2017; 23: 1598-606.

13. Singhi AD, Liu TC, Roncaioli JL, et al. Alternative lengthening of telomeres and loss of DAXX/ATRX expression predicts metastatic disease and poor survival in patients with pancreatic neuroendocrine Tumors. Clin Cancer Res 2017; 23: 600-9.

14. Kim JY, Kim MS, Kim KS, et al. Clinicopathologic and prognostic significance of multiple hormone expression in pancreatic neuroendocrine tumors. Am J Surg Pathol 2015; 39: 592-601.

15. Arnason T, Sapp HL, Barnes PJ, Drewniak M, Abdolell M, Rayson D. Immunohistochemical expression and prognostic value of ER, PR and HER2/neu in pancreatic and small intestinal neuroendocrine tumors. Neuroendocrinology 2011; 93: 249-58.

16. Viale G, Doglioni C, Gambacorta M, Zamboni G, Coggi G, Bordi C. Progesterone receptor immunoreactivity in pancreatic endocrine tumors: an immunocytochemical study of 156 neuroendocrine tumors of the pancreas, gastrointestinal and respiratory tracts, and skin. Cancer 1992; 70: 2268-77.

17. Doglioni C, Gambacorta M, Zamboni G, Coggi G, Viale G. Immunocytochemical localization of progesterone receptors in endocrine cells of the human pancreas. Am J Pathol 1990; 137: 999-1005.

18. Hruban RH, Pitman MB, Klimstra DS. Tumors of the pancreas. Washington, DC: American Registry of Pathology, 2007.

19. Tang LH, Gonen M, Hedvat C, Modlin IM, Klimstra DS. Objective quantification of the Ki67 proliferative index in neuroendocrine tumors of the gastroenteropancreatic system: a comparison of digital image analysis with manual methods. Am J Surg Pathol 2012; 36: 1761-70.

20. Son EM, Kim JY, An S, et al. Clinical and prognostic significances of cytokeratin 19 and KIT expression in surgically resectable pancreatic neuroendocrine tumors. J Pathol Transl Med 2015; 49: 30-6.

21. Roh J, Knight S, Chung JY, et al. S100A4 expression is a prognostic indicator in small intestine adenocarcinoma. J Clin Pathol 2014; 67: 216-21.

22. Jang KT, Park SM, Basturk O, et al. Clinicopathologic characteristics of 29 invasive carcinomas arising in 178 pancreatic mucinous cystic neoplasms with ovarian-type stroma: implications for management and prognosis. Am J Surg Pathol 2015; 39: 179-87.

23. Anlauf M, Perren A, Klöppel G. Endocrine precursor lesions and microadenomas of the duodenum and pancreas with and without MEN1: criteria, molecular concepts and clinical significance. Pathobiology 2007; 74: 279-84.

24. Anlauf M, Schlenger R, Perren A, et al. Microadenomatosis of the endocrine pancreas in patients with and without the multiple endocrine neoplasia type 1 syndrome. Am J Surg Pathol 2006; 30: 560-74.

25. Hackeng WM, Brosens LA, Poruk KE, et al. Aberrant Menin expression is an early event in pancreatic neuroendocrine tumorigenesis. Hum Pathol 2016; 56: 93-100.

26. Hadano A, Hirabayashi K, Yamada M, et al. Molecular alterations in sporadic pancreatic neuroendocrine microadenomas. Pancreatology 2016; 16: 411-5.

27. de Wilde RF, Heaphy CM, Maitra A, et al. Loss of ATRX or DAXX expression and concomitant acquisition of the alternative lengthening of telomeres phenotype are late events in a small subset of MEN-1 syndrome pancreatic neuroendocrine tumors. Mod Pathol 2012; 25: 1033-9.

28. Estrella JS, Broaddus RR, Mathews A, et al. Progesterone receptor and PTEN expression predict survival in patients with low- and intermediate-grade pancreatic neuroendocrine tumors. Arch Pathol Lab Med 2014; 138: 1027-36.

29. Pelosi G, Bresaola E, Bogina G, et al. Endocrine tumors of the pancreas: Ki-67 immunoreactivity on paraffin sections is an independent predictor for malignancy: a comparative study with proliferating-cell nuclear antigen and progesterone receptor protein immunostaining, mitotic index, and other clinicopathologic variables. Hum Pathol 1996; 27: 1124-34.

30. Li X, O'Malley BW. Unfolding the action of progesterone receptors. J Biol Chem 2003; 278: 39261-4.

31. Yazdani S, Kasajima A, Ogata H, et al. Progesterone receptor isoforms $A$ and $B$ in pancreatic neuroendocrine tumor. Neuroendocrinology 2015; 101: 309-20. 